University of Wollongong

Research Online

Australian Institute for Innovative Materials -

Papers

Australian Institute for Innovative Materials

$1-1-2014$

Microscopic model for exchange bias from grain-boundary disorder in a ferromagnet/antiferromagnet thin film with a nanocrystalline microstructure

David L. Cortie

University of Wollongong, dlc422@uowmail.edu.au

A G. Biternas

University of York

R W. Chantrell

University of York

Xiaolin Wang

University of Wollongong, xiaolin@uow.edu.au

Frank Klose

ANSTO, Australian Nuclear Science And Technology Organisation, Frank.Klose@ansto.gov.au

Follow this and additional works at: https://ro.uow.edu.au/aiimpapers

Part of the Engineering Commons, and the Physical Sciences and Mathematics Commons

Research Online is the open access institutional repository for the University of Wollongong. For further information contact the UOW Library: research-pubs@uow.edu.au 


\title{
Microscopic model for exchange bias from grain-boundary disorder in a ferromagnet/antiferromagnet thin film with a nanocrystalline microstructure
}

\begin{abstract}
Monte Carlo spin simulations were coupled to a Voronoi microstructure-generator to predict the magnitude and behavior of exchange bias in a ferromagnet/antiferromagnet (AF) thin film bilayer with a nanocrystalline microstructure. Our model accounts for the effects of irregular grain-shapes, finite-sized particles, and the possible presence of local random-fields originating from the antiferromagnet's grainboundary regions. As the grain-boundary represents a crystal-structure distortion, we model the local effect on the exchange constants in the Gaussian approximation which can cause regions resembling a spin glass confined to an unusual 2D topology. Although an ensemble of completely disconnected AF grains isolated by non-magnetic barriers provides a small exchange bias, the introduction of a spin-glass network at the boundaries causes a four-fold enhancement in the magnitude of the loop-shift. This implies the importance of local grain-boundary behavior in defect-engineered antiferromagnets.
\end{abstract}

\section{Keywords}

bias, grain, boundary, disorder, ferromagnet, antiferromagnet, thin, film, model, nanocrystalline, microscopic, microstructure, exchange

\section{Disciplines}

Engineering | Physical Sciences and Mathematics

\section{Publication Details}

Cortie, D. L., Biternas, A. G., Chantrell, R. W., Wang, X. L. \& Klose, F. (2014). Microscopic model for exchange bias from grain-boundary disorder in a ferromagnet/antiferromagnet thin film with a nanocrystalline microstructure. Applied Physics Letters, 105 (3), 032402-1-032402-5. 


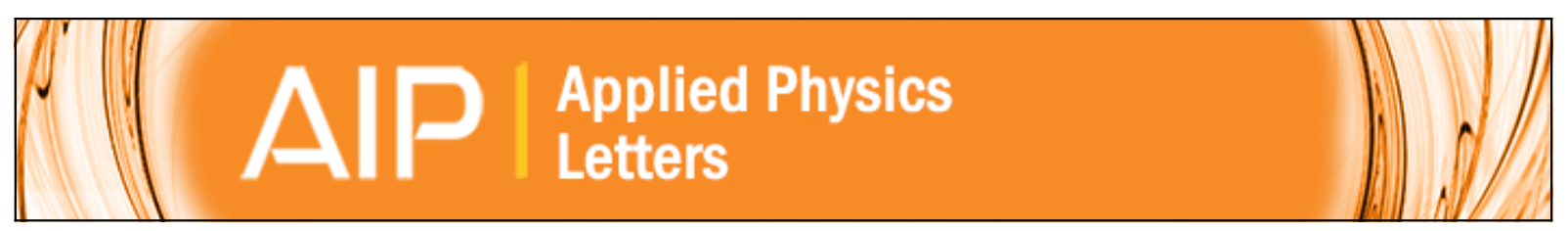

\section{Microscopic model for exchange bias from grain-boundary disorder in a ferromagnet/antiferromagnet thin film with a nanocrystalline microstructure}

D. L. Cortie, A. G. Biternas, R. W. Chantrell, X. L. Wang, and F. Klose

Citation: Applied Physics Letters 105, 032402 (2014); doi: 10.1063/1.4890580

View online: http://dx.doi.org/10.1063/1.4890580

View Table of Contents: http://scitation.aip.org/content/aip/journal/apl/105/3?ver=pdfcov

Published by the AIP Publishing

\section{Articles you may be interested in}

Seeking to quantify the ferromagnetic-to-antiferromagnetic interface coupling resulting in exchange bias with various thin-film conformations

J. Appl. Phys. 116, 053911 (2014); 10.1063/1.4892177

Exchange bias effect in Ti doped nanocrystalline SrFeO3-

AIP Advances 4, 087144 (2014); 10.1063/1.4894486

Effect of antiferromagnetic layer thickness on exchange bias, training effect, and magnetotransport properties in ferromagnetic/antiferromagnetic antidot arrays

J. Appl. Phys. 115, 133909 (2014); 10.1063/1.4870285

Exchange bias and its thermal stability in ferromagnetic/antiferromagnetic antidot arrays Appl. Phys. Lett. 101, 012407 (2012); 10.1063/1.4733341

Field-induced transitions from negative to positive exchange bias in nanoparticles with inverted ferromagneticantiferromagnetic core-shell morphology

J. Appl. Phys. 111, 053904 (2012); 10.1063/1.3688352

AlP $\left.\right|_{\text {Letters }} ^{\text {Applied Physics }}$

is pleased to announce Reuben Collins as its new Editor-in-Chief

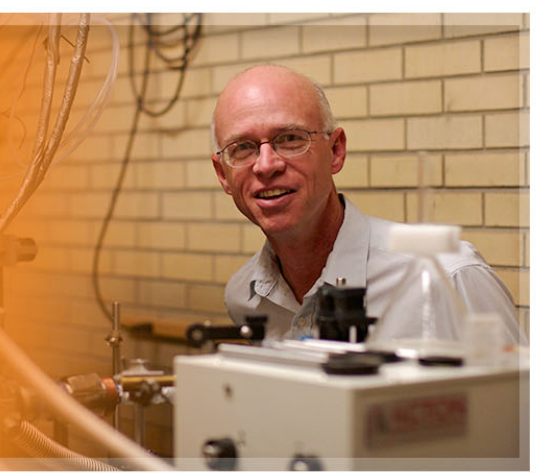




\title{
Microscopic model for exchange bias from grain-boundary disorder in a ferromagnet/antiferromagnet thin film with a nanocrystalline microstructure
}

\author{
D. L. Cortie, ${ }^{1,2,3}$ A. G. Biternas, ${ }^{4}$ R. W. Chantrell, ${ }^{4}$ X. L. Wang, ${ }^{1}$ and F. Klose ${ }^{2,5}$ \\ ${ }^{1}$ The Institute for Superconducting and Electronic Materials, The University of Wollongong, Wollongong, \\ NSW 2522, Australia \\ ${ }^{2}$ Australian Nuclear Science and Technology Organisation, Lucas Heights, NSW 2234, Australia \\ ${ }^{3}$ Quantum Matter Institute, University of British Columbia, Vancouver, British Columbia V6T 1Z4, Canada \\ ${ }^{4}$ Department of Physics, University of York, YO10 5DD, United Kingdom \\ ${ }^{5}$ Department of Physics and Materials Science, City University of Hong Kong, Hong Kong, China
}

(Received 29 April 2014; accepted 4 July 2014; published online 22 July 2014)

\begin{abstract}
Monte Carlo spin simulations were coupled to a Voronoi microstructure-generator to predict the magnitude and behavior of exchange bias in a ferromagnet/antiferromagnet (AF) thin film bilayer with a nanocrystalline microstructure. Our model accounts for the effects of irregular grain-shapes, finite-sized particles, and the possible presence of local random-fields originating from the antiferromagnet's grain-boundary regions. As the grain-boundary represents a crystal-structure distortion, we model the local effect on the exchange constants in the Gaussian approximation which can cause regions resembling a spin glass confined to an unusual 2D topology. Although an ensemble of completely disconnected AF grains isolated by non-magnetic barriers provides a small exchange bias, the introduction of a spin-glass network at the boundaries causes a four-fold enhancement in the magnitude of the loop-shift. This implies the importance of local grain-boundary behavior in defect-engineered antiferromagnets. (C) 2014 AIP Publishing LLC.
\end{abstract}

[http://dx.doi.org/10.1063/1.4890580]

Antiferromagnetism is a common state of spin order in condensed matter; however, the interfacial behavior near a break in translational symmetry is challenging to understand owing to the plethora of additional effects that emerge. ${ }^{1-4}$ Thin film antiferromagnets form a core component in contemporary data storage, ${ }^{4}$ and are poised to play a unique role in the next generation of spin-based electronics. ${ }^{5-7}$ In particular, the ability to induce an effective unidirectional anisotropy known as exchange bias (EB) using an antiferromagnet (AF) coupled to a ferromagnet (FM) in various nanoarchitectures provides a crucial functionality to design ${ }^{5}$ and switch $^{6,7}$ spin structures at atomic scales. Macroscopically, exchange bias is well known to lead to shifts of the ferromagnet hysteresis loop and an increase in coercivity. ${ }^{2}$ The precise microscopic mechanisms for these symptoms, however, have remained heavily debated. ${ }^{3,4}$ Here, we present a model for the microscopic formation of a weak net ferromagnetic moment responsible for exchange bias in nanocrystalline (NC) antiferromagnets. Past models have explained the source of similar surface moments in specific cases such as epitaxial films due to natively uncompensated surfaces, ${ }^{3}$ interface roughness, ${ }^{8}$ or magnetization from antiferromagnetic domain walls. ${ }^{9}$ Prior work has emphasized the magnetic role of the single interface at the junction between the FM and AF, whereas less consideration has been given to the possible presence of multiple interfaces within the antiferromagnet itself. This is crucial for nanocrystalline AFs, which are structurally characterized by grain sizes in the $1-250 \mathrm{~nm}$ range and possess a large volume fraction $(\leq 50 \%)$ of atoms positioned at grainboundary interfaces. ${ }^{10,11}$ Grain boundaries typically consist of dislocations or amorphous regions which dramatically modify properties such as hardness, specific-heat, and diffusivity. ${ }^{10,11}$ It is well-accepted that EB is often maximized in FM/AF films using a nanocrystalline AF (Ref. 2) rather than chemically similar epitaxial films. The maximum EB field $H_{E B}$ scales inversely with AF grain size in polycrystalline $\mathrm{NiO}$ and $\mathrm{CoO}$ (Refs. 12 and 13) and this is connected to a similar relationship between the AF domain size and exchange bias in epitaxial films. ${ }^{14,15}$ Contemporary industrial spin valves rely on antiferromagnetic materials with small grains $(5-30 \mathrm{~nm}){ }^{4}$ Given that grain morphology is a key factor influencing EB-behavior in this important material class, ${ }^{3,4}$ it seems necessary to revisit the contemporary microscopic models to re-examine the effect of disorder in the context of nanocrystalline antiferromagnets.

For isolated AF nanometer-sized particles, Néel predicted an uncompensated moment expected from the finitesize constraint preventing the cancellation of staggered magnetization. ${ }^{1}$ In interconnected nanograins, however, there is a possibility of a modified boundary exchange interaction. ${ }^{16}$ Using geometric atomistic arguments, Takano et al. calculated the density of uncompensated moments in an elliptical AF grain showing these scaled as $L^{-1}$, where $L$ is the characteristic diameter of the grain. ${ }^{12}$ Other models used rectangular grains of uniform size ${ }^{17}$ or treated the spins in a grain particle as a collective entity. ${ }^{18-21}$ All of this pioneering work, however, made assumptions: that antiferromagnetic order was perfectly disrupted at a grain-boundary; ${ }^{12}$ that grains form well-ordered arrays with regularly shaped boundaries; ${ }^{18}$ or that the net $\mathrm{AF}$ surface magnetization remains irreversible during the magnetic hysteresis. ${ }^{12}$ Here, we shall explore the consequences of relaxing these assumptions. Our main proposal is that the modifications at a grainboundary are intimately linked to the properties of the local 
magnetic network. The structural effects at a grain boundary are diverse, as is their effect on the magnetic environment. High-resolution microscopy shows modified local bonding angles ${ }^{16}$ and variance in interatomic separation. ${ }^{22}$ Based on the general understanding of amorphous magnets, we argue that these features preserve the magnetic moment but introduce disorder into the exchange coupling integrals. ${ }^{16}$ In such cases, the molecular fields at the grain boundaries may be "damaged" to follow a random distribution resulting from the disorganized local environment interrupting $\mathrm{AF}$ order to a variable degree ${ }^{16,23-25}$ analogous to boundaries in ferromagnets. ${ }^{26}$ As the local magnetic effects of an irregular grain-boundary interface have not been considered in past microscopic models, existing theories invoke a phenomenological parameter termed the "contact fraction" $C^{*}$, intrinsic bias $H_{e}^{i}$, or effective interfacial coupling distribution to explain the correct magnitude of EB. ${ }^{4,19,27}$ Such parameters are difficult to predict, because they involve the net effect of uncompensated surface $\mathrm{AF}$ spins due to specific grain orientations ${ }^{12}$ along with emergent magnetic behavior from interfacial roughness, defects, and grain-boundaries. ${ }^{27}$ In this work, we simulate NC ferromagnetic/antiferromagnetic layered films with a 3D atomic-scale spin model using only reasonable values of the fundamental exchange integrals as input. This is shown to produce the correct order of magnitude of EB without further assumptions. For the sake of completeness, we considered both magnetic and non-magnetic grain boundaries. The wide parameter space where our model produces exchange bias offers a satisfying explanation for why EB is ubiquitous in nanocrystalline AFs.

To generate the nanocrystalline microstructure, we employed the standard algorithm for Voronoi tessellation which mimics a process of crystallization where a number of grains simultaneously nucleate and expand isotropically at an identical rate. ${ }^{28}$ This method approximates a realistic grain distribution for thin film materials on different types of lattices, ${ }^{29}$ and has been used for mechanical stress calculations ${ }^{28,30}$ and to model NC ferromagnets. ${ }^{26}$ The overall distribution depends on the number of grain centers which is expressed as the nucleation density $\rho_{V}$, which is the fraction of randomly distributed sites that behave as nucleation points. Each spin is assigned to belong to the grain linked by the nearest common nucleation point. ${ }^{31}$ Although the geometric construction is unique for each random seed, the average distribution resembles the common experimental form $^{4}$ and can be fitted with a log-normal function $f(x)=A_{0} \exp \left(-\left[\frac{\ln \left(x / x_{0}\right)}{w}\right]^{2}\right)$, where $A_{0}$ is the mode of the distribution, $x_{0}$ is related to the mean of the distribution, and $w$ is the width parameter. The fits to the histograms generated from averaging over ten random seeds are shown in Fig. 1, and the results are summarized in the inset, along with a plane-view image of the grain structure on the $128 \times 128$ cubic spin lattice, viewed along the [100] direction normal to the film plane. After constructing the microstructure, defects are placed at the boundaries dividing each grain.

To account for spin behavior in proximity to the defect wall separating two grains, we adopt a Hamiltonian closely based on the Domain State Model $^{9}$ consisting of a FM coupled to a disordered AF

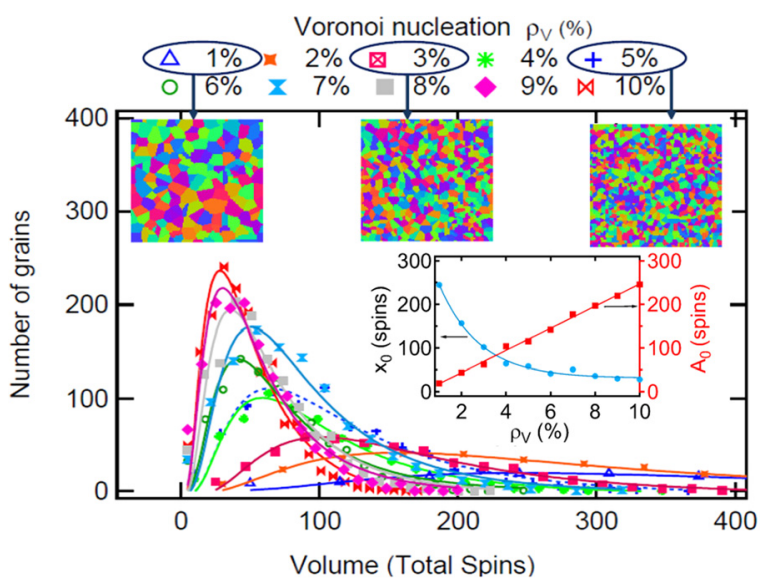

FIG. 1. Histogram of grain volumes for different nucleation percentage averaged over 10 simulations. Solid lines are a fit to the data with a lognormal function. The insets show the fitting results and the simulated grain structure on the $128 \times 128$ cubic lattice.

$$
\begin{aligned}
\mathcal{H}= & -J_{\mathrm{FM}} \sum_{i, j \in \mathrm{FM}} \vec{S}_{i} \cdot \vec{S}_{j}-\sum_{i \in \mathrm{FM}}\left(K_{\mathrm{FM}}^{s} S_{i x}^{2}+K_{\mathrm{FM}}^{u} S_{i z}^{2}+\mu \vec{B}_{e x t} \cdot \vec{S}_{i}\right) \\
& -\sum_{i, j \in \mathrm{AF}} J_{\mathrm{AF}}^{i j} \vec{S}_{i} \cdot \vec{S}_{j}-\sum_{i \in \mathrm{AF}}\left(K_{\mathrm{AF}}^{u} S_{i z}^{2}+\mu \vec{B}_{e x t} \cdot \vec{S}_{i}\right) \\
& -\sum_{i \in \mathrm{AF}, j \in \mathrm{FM}} J_{i n t}^{i j} \vec{S}_{i} \cdot \vec{S}_{j}
\end{aligned}
$$

The summations are performed on a simple cubic lattice considering only the exchange coupling with the six cubicnearest neighbors. The first line in Eq. (1) describes a FM with total spin $\left|\vec{S}_{\mathrm{i}}\right|=1$ (and total magnetic moment $\mu$ ), ferromagnetic exchange $J_{\mathrm{FM}}=1$, uniaxial anisotropy $K_{\mathrm{FM}}^{u}$ $=0.1 J_{\mathrm{FM}}$, and shape anisotropy $K_{\mathrm{FM}}^{s}=-0.1 J_{\mathrm{FM}}$. The second line considers a disordered AF with $\left|\vec{S}_{\mathrm{i}}\right|=1$ and $J_{\mathrm{AF}}^{i j}=-0.5 J_{\mathrm{FM}}$ (when neither $i$ nor $j$ are defects). The third line describes a ferromagnetic interaction between FM and $\mathrm{AFM}$ in the interface with $J_{i n t}^{i j}=0.5 J_{\mathrm{FM}}$.

Grain-boundary sites in the AF lattice are replaced by defects where $J_{\mathrm{AF}}^{i j}$ and $S_{\mathrm{i}}$ is modified in various ways according to the distributions specified in Fig. 2. We investigate four possible types of magnetic grain boundaries: non-magnetic, modulated, weakened, or glassy exchange networks. For the first case, $\left|\vec{S}_{i}\right|=0.0$ and $J_{\mathrm{AF}}^{i j}=0$ (when $i$ is a defect site), whereas for the other scenarios $\left|\overrightarrow{S_{i}}\right|=1$ but the local value of $J_{\mathrm{AF}}^{i j}$ is a random variable drawn from a Gaussian distribution

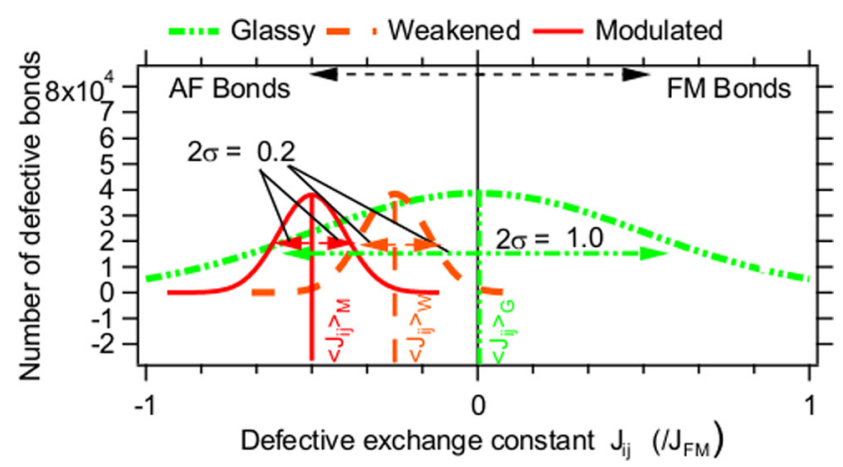

FIG. 2. Parameters used in the exchange bias simulation to model different types of spin behavior at the AF grain-boundary when $i$ is a defect spin, $\left\langle J^{i j}\right\rangle$ is the mean, and $\sigma$ is the standard deviation of the Gaussian distribution. 
which retains a constant value set at the beginning of the simulation, mimicking the effect of quenched disorder associated with a variable structural environment. The grain-boundary is taken to be one atomic spin thick. The bilayer consists of a single FM monolayer $\left(t_{\mathrm{FM}}=1\right)$ in contact with four layers of Ising $\mathrm{AF}$ material $\left(t_{\mathrm{AF}}=4\right)$ with a lateral dimension of $128 \times 128$ so that a total of 81920 spins were included. Periodic boundary conditions were applied in the lateral dimension. Here, we model the AF as a system having high anisotropy (Ising) since the effect of random-anisotropy has been studied before, ${ }^{17,18,32}$ but it is necessary to isolate the specific effects of intra-antiferromagnet grain-boundary exchange. We assume that the spins in the grain boundaries couple equally to the FM so that $J_{\text {int }}^{\text {grain }}=J_{\text {int }}^{\text {bulk }}$. In the current work, the FM is of Heisenberg-type and was approximated as a single-grain entity. Throughout later sections, the reduced temperature $\tau$ is expressed in natural units of the strongest exchange energy $\left(J_{\mathrm{FM}}\right)$ such that $\tau=k_{B} T / J_{\mathrm{FM}}$ and the field is written in reduced form $b=\mu B_{\text {ext }}$ in units of the FM exchange parameter $J_{\mathrm{FM}}$. To simulate field-cooling, the sample is initialized in the paramagnetic state $(\tau>1.45)$ and cooled to $\tau=0.1$ in a saturating field along the in-plane $\hat{z}$ direction with magnitude $b=0.2$ taking $10^{3}$ Monte-Carlo steps (total steps per spin) per 0.1 temperature interval. A trial step involves a small rotation of the spin around the unit sphere for the Heisenberg spins or a spin-flip in $\hat{z}$ for the Ising spins. A standard heatbath algorithm is used for accepting moves. ${ }^{33}$ At $\tau=0.1$, the field is swept between $b=-0.25$ and $b=0.25$ to simulate a hysteresis loop in a similar method to Ref. 9. The EB field is $b_{\mathrm{EB}}=\left(b_{c 1}+b_{c 2}\right) / 2$, where $b_{c 1}$ and $b_{c 2}$ are the first and second coercive fields.

Fig. 3(a) illustrates typical magnetic hysteresis loops for the FM obtained at $\tau=0.1$, where different AF grainboundary behaviors were adopted for identical geometrical grain distributions $\left(\rho_{V}=0.05\right)$. In all cases, an EB field is evident in the FM and, by comparing the magnetic hysteresis loops of the AF layers in Figs. 3(b)-3(d), it is clear that the grain boundaries greatly influence the AF response. Fig. 3(b) illustrates a typical AF hysteresis loop obtained for the nonmagnetic boundary. The coercivity/saturation and vertical shift in the AF imply a small rotatable interfacial moment of $\approx 0.5 \% M_{\text {sat }}$ and frozen interfacial moment of $\approx 0.75 \% M_{\text {sat }}$, respectively. Fig. 3(c) shows the case for a modulated boundary. The AF obtains a frozen moment of $1 \%$ and rotatable moment of $3.5 \%$. For the weakened grain-boundary shown in Fig. 3(d), an increased paramagnetic signal originates from the interface evident in the non-saturating hysteresis loop as a result of more spins with low $J_{\mathrm{AF}}^{i j} \cdot{ }^{37}$ This does not result in a larger $\mathrm{EB}$, as the irreversible component is small $\left(<1 \% M_{\text {sat }}\right)$. Fig. 3(e) is an example of the glassy type of grain-boundary providing a larger bias than the other scenarios. The vertical shift of the bulk antiferromagnetic spins is the largest $\left(10 \% M_{\text {sat }}\right)$ and the interfacial regions are highly polarized (reaching approximately $30 \%$ of their "ferromagnetic" arrangement). The relaxation of magnetization on the time-scales of the measurement causes a failure to obtain a closed loop which is a precondition for the magnetic training effect.

Fig. 4 summarizes the dependence of the EB field and coercivity for the various grain-boundary types versus the
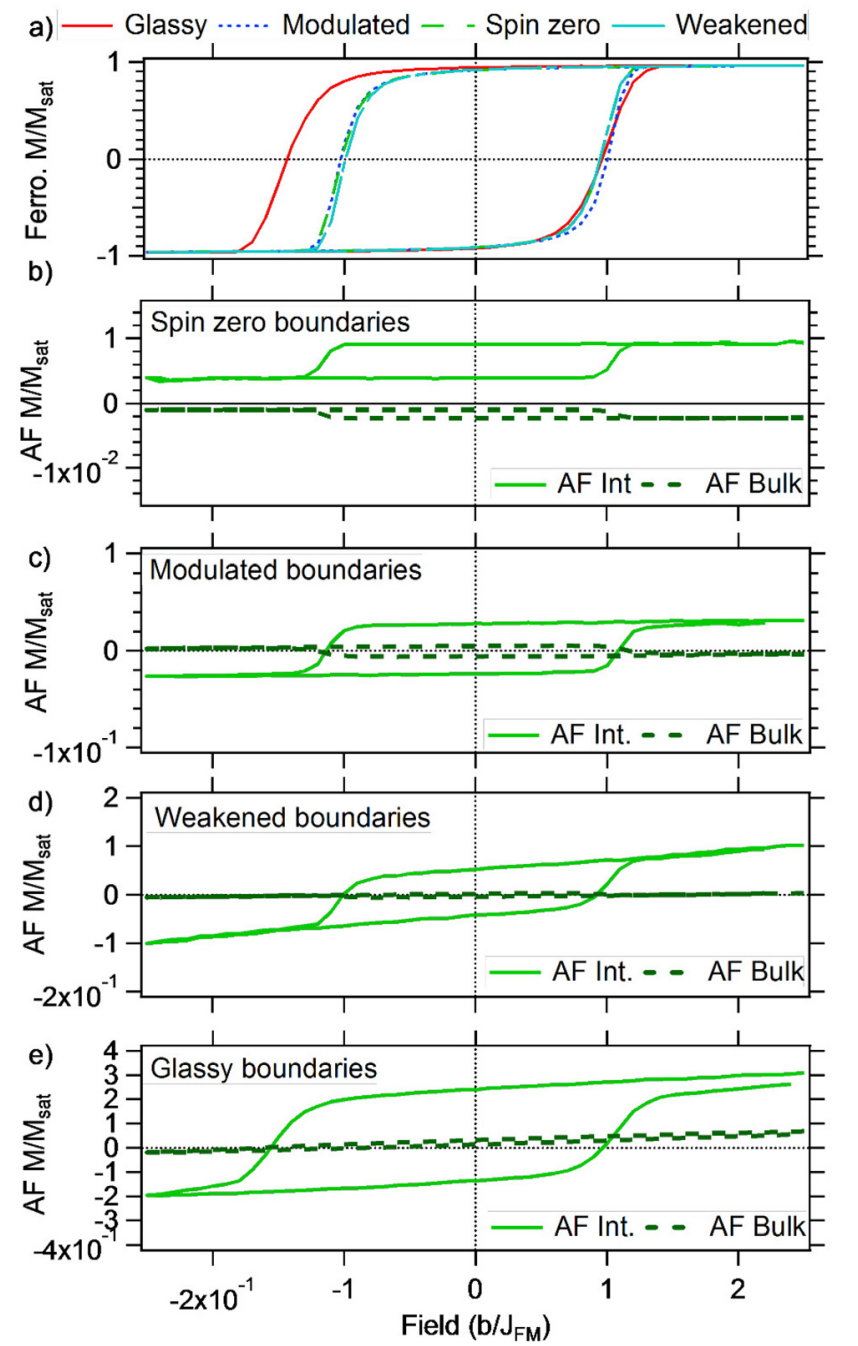

FIG. 3. Hysteresis loops of the (a) FM and AF for (b) spin zero, (c) glassy, (d) modulated, and (e) weakened grain-boundary types in the field-cooled state.

equivalent average grain diameter calculated from the grain surface area based on the fitted parameters from Fig. 1 simulations with different $\rho_{V}$. Each data point have been averaged over ten random microstructural seeds to reduce statistical error. Remarkably, Fig. 4 shows that, for all boundary types, the EB scales approximately as $L^{-1}$ in accordance with Takano's results for CoO (Ref. 12) although our model assumes a very different, flat [100] surface that is nominally
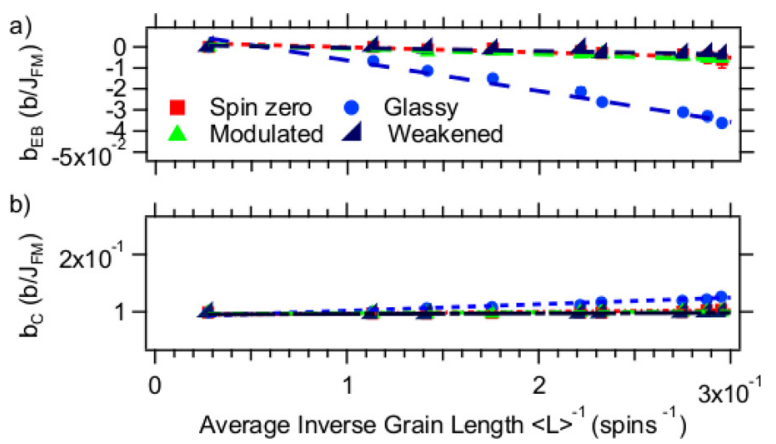

FIG. 4. (a) Exchange bias and (b) coercivity dependency on inverse mean grain size showing approximately linear scaling with different types of grain-boundary defect behavior. The lines are guides for the eye. 


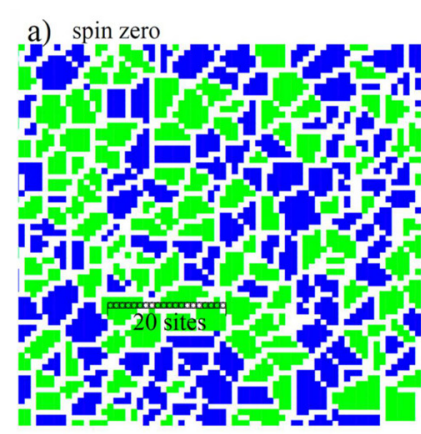

AF Grain Phase 1

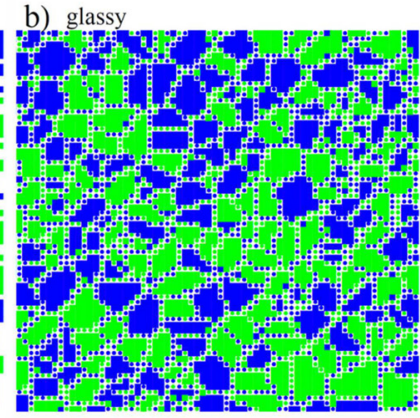

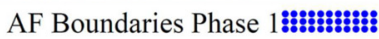
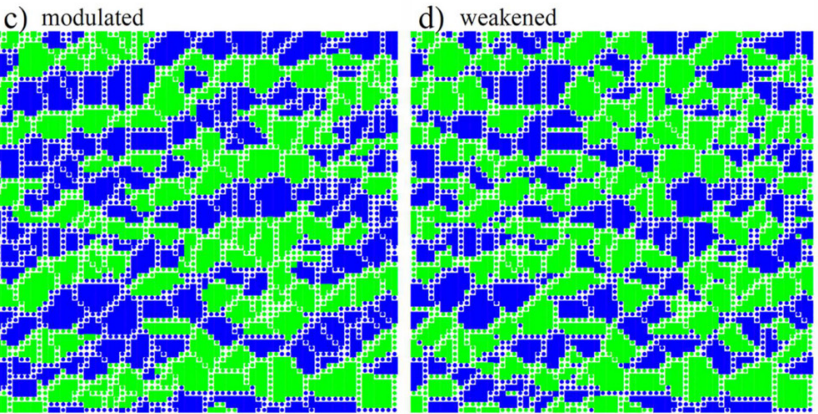

AF Grain Phase 2

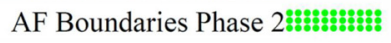

FIG. 5. Snapshots of the AF interfacial spin structure showing domain distribution for the two AF domain phases (green/blue) after field-cooling for (a) spin zero, (b) glassy, (c) modulated, and (d) weakened grain-boundary types.

compensated. This suggests that grain-geometry is a dominant force affecting the relative strength of $\mathrm{EB}$, whereas the overall magnitude is set by the details of the grain-boundary. Experimentally it is known that patterning continuous AF/FM films into nanodots cuts the grain distribution and introduces new contributions to exchange bias from edge-effects. ${ }^{34}$ In direct connection to experiment, our simulation implies that a frustrated AF structure at an irregular grain edge provides a contribution to bias. ${ }^{34}$

The peak magnitude of EB for the non-magnetic, modulated, and weakened grain-boundary types is $\mathrm{b}_{\mathrm{EB}} \approx 0.01 / J_{\mathrm{FM}}$, which is the correct order of magnitude for experiment. ${ }^{9} \mathrm{On}$ the other hand, the EB for the glassy-exchange is four times larger $b_{\mathrm{EB}} \approx 0.04 / J_{\mathrm{FM}}$. Despite the zero average exchange across the glassy boundary, the wide variance includes the possibility of ferromagnetic bonds which greatly assist the formation of EB. This concurs with a previous theoretical study which showed a much larger EB for a Gaussian spin-glass. ${ }^{35}$ Significant EB has been seen in spin-glasses experimentally. ${ }^{36}$ As seen in Fig. 4(b), coercivity increases slightly for the non-magnetic, modulated, and weakened grain-boundary types and scales approximately as $L^{-1}$. This correlates with the increasing number of rotatable $\mathrm{AF}$ interfacial moments. The large linear increase of coercivity for the glassy boundary implies that the mixture of ferromagnetic and antiferromagnetic interactions induce a larger anisotropy. This is analogous to model EB systems with rough interfaces, ${ }^{8}$ where rotable AFM spins are embedded in the interfacial matrix of FM spins (or vice versa) yielding an induced anisotropy.

Direct examination of the AF domain structure using $\mathrm{X}$-ray techniques may allow experimentalists to deduce the types of boundaries in specific materials. ${ }^{15}$ Fig. 5 shows a picture of $\mathrm{AF}$ phase domain structure in a section of the $128 \times 128(\approx 60 \times 60 \mathrm{~nm})$ simulated film plane after fieldcooling for the four different types of grain boundaries at $\rho_{V}=0.05$. Analysis of the AF phase domains for the nonmagnetic boundary in Fig. 5(a) shows that the majority of particles possess a single AF domain, and domain walls do not traverse the grain-boundary. The AF is ordered within each grain, but neighboring grains may be out-of-phase with one other. A similar situation is apparent for the glassy type of boundary where $\left\langle J_{i j}\right\rangle=0$, although regions of the grain boundaries possess short-range ferromagnetic order apparent in the high density of point defects in the $\mathrm{AF}$ order parameter. By contrast, a long-range domain state is realized in weakened and modulated grain boundaries in Figs. 5(c) and 5(d), with domain walls that are weakly pinned to the grain-boundaries but may also cross them. The domain structure is similar to the standard Domain State model. ${ }^{9}$

The main conclusion of this work is that grain-boundary defects can enhance the weak net magnetic moment at the surface of an antiferromagnetic layer. Terminating the antiferromagnetic domain at a boundary encourages the production of uncompensated moments. For statistical reasons, this can occur even for completely decoupled grains. However, in the case of randomly coupled grains, there is still an energetic advantage to forming a domain state due to random-field point defects. ${ }^{9}$ Our model reproduces the relation between $\mathrm{AF}$ domain-size and $\mathrm{EB}$, as observed experimentally, ${ }^{15}$ and confirms the importance of small antiphasedomains in producing an uncompensated moment such as that detected with atomic-scale microscopy. ${ }^{38}$ In our model, EB scales approximately as $L^{-1}$ with no apparent limit. ${ }^{12,13}$ Future investigations are needed to establish whether larger grains are required to stabilize to EB in low anisotropy antiferromagnets as suggested, for example, in Ref. 4. Controlling magnetic grain-boundary behavior in nanocrystalline materials may lead to defect-engineering of other unique and previously unanticipated properties.

David Cortie acknowledges the support of the Australian Institute of Nuclear Science and Engineering.

${ }^{1}$ N. Kurti, Selected Works of Louis Neel (Taylor \& Francis, New York, 1998).

${ }^{2}$ J. Nogues and I. K. Schuller, J. Magn. Magn. Mater. 192, 203 (1999).

${ }^{3}$ R. L. Stamps, J. Phys. D: Appl. Phys. 33, R247 (2000).

${ }^{4}$ K. O'Grady, L. E. Fernandez-Outon, and G. Vallejo-Fernandez, J. Magn. Magn. Mater. 322, 883 (2010).

${ }^{5}$ B. G. Park, J. Wunderlich, X. Marti, V. Holy, Y. Kurosaki, M. Yamada, H. Yamamoto, A. Nishide, J. Hayakawa, H. Takahashi, A. B. Shick, and T. Jungwirth, Nat. Mater. 10, 347 (2011).

${ }^{6}$ X. He, Y. Wang, N. Wu, A. N. Caruso, E. Vescovo, K. D. Belashchenko, P. A. Dowben, and C. Binek, Nat. Mater. 9, 579 (2010).

${ }^{7}$ Y.-H. Chu, L. W. Martin, M. B. Holcomb, M. Gajek, S.-J. Han, Q. He, N. Balke, C.-H. Yang, D. Lee, W. Hu, Q. Zhan, P.-L. Yang, A. FraileRodríguez, A. Scholl, S. X. Wang, and R. Ramesh, Nat. Mater. 7, 478 (2008).

${ }^{8}$ J. Spray and U. Nowak, J. Phys. D: Appl. Phys. 39, 4536 (2006).

${ }^{9}$ U. Nowak, K. D. Usadel, J. Keller, P. Miltényi, B. Beschoten, and G. Güntherodt, Phys. Rev. B 66, 014430 (2002).

${ }^{10}$ H. Gleiter, Prog. Mater. Sci. 33, 223 (1989).

${ }^{11}$ M. Meyers, A. Mishra, and D. Benson, Prog. Mater. Sci. 51, 427 (2006). 
${ }^{12}$ K. Takano, R. H. Kodama, A. E. Berkowitz, W. Cao, and G. Thomas, Phys. Rev. Lett. 79, 1130 (1997).

${ }^{13}$ C-H. Nam, B. K. Cho, and S. Lee, J. Appl. Phys. 93, 6584 (2003).

${ }^{14}$ H. Béa, M. Bibes, F. Ott, B. Dupé, X.-H. Zhu, S. Petit, S. Fusil, C. Deranlot, K. Bouzehouane, and A. Barthélémy, Phys. Rev. Lett. 100, 017204 (2008).

${ }^{15}$ A. Scholl, F. Nolting, J. W. Seo, H. Ohldag, J. Stöhr, S. Raoux, J.-P. Locquet, and J. Fompeyrine, Appl. Phys. Lett. 85, 4085 (2004).

${ }^{16}$ D. T. Margulies, F. T. Parker, M. L. Rudee, F. E. Spada, J. N. Chapman, P. R. Aitchison, and A. E. Berkowitz, Phys. Rev. Lett. 79, 5162 (1997).

${ }^{17}$ D. Suess, T. Schrefl, W. Scholz, J.-V. Kim, R. L. Stamps, and J. Fidler, IEEE Trans. Magn. 38, 2397 (2002).

${ }^{18}$ M. D. Stiles and R. D. McMichael, Phys. Rev. B 59, 3722 (1999).

${ }^{19}$ M. Gruyters, Phys. Rev. B 79, 134415 (2009).

${ }^{20}$ D. Suess, M. Kirschner, T. Schrefl, J. Fidler, R. L. Stamps, and J.-V. Kim, Phys. Rev. B 67, 054419 (2003).

${ }^{21}$ A. Harres and J. Geshev, J. Phys.: Condens. Matter 24, 326004 (2012).

${ }^{22}$ D. Williams and C. Carter, Transmission Electron Microscopy: A Textbook for Material Scientists (Plenum Press, New York, 1996), pp. 5-10.

${ }^{23}$ A. Hernando, J. Phys.: Condens. Matter. 11, 9455 (1999).

${ }^{24}$ P. G. Bercoff and H. R. Bertorello, Appl. Phys. A 100, 1019 (2010).

${ }^{25}$ R. B. Gangineni, K. Dör, N. Kozlova, K. Nenkov, K.-H. Müller, L. Schultz, and L. S. Lakshmi, J. Appl. Phys. 99, 053904 (2006).

${ }^{26}$ G.-P. Zheng, D. Gross, and M. Li, J. Appl. Phys. 93, 7652 (2003).
${ }^{27}$ V. Baltz, B. Rodmacq, A. Zarefy, L. Lechevallier, and B. Dieny, Phys. Rev. B 81, 052404 (2010).

${ }^{28}$ R. Quey, P. Dawson, and F. Barbe, Comput. Meth. Appl. Mech. Eng. 200, 1729 (2011).

${ }^{29}$ D. Gross and M. Li, Appl. Phys. Lett. 80, 746 (2002).

${ }^{30}$ J. Schiøtz, T. Vegge, F. D. di Tolla, and K. W. Jacobsen, Phys. Rev. B 60, 11971 (1999), e-print arXiv:cond-mat/9812102.

${ }^{31} \mathrm{To}$ form columnar grains, the distance operator $d_{i j}\left(S_{i}, S_{j}\right)=$ $\sqrt{\left(r_{i y}-r_{j y}\right)^{2}+\left(r_{i z}-r_{j z}\right)^{2}}$ is used in the tesselation to form in-plane 2D polyhedral extruded in the $\hat{x}$ direction mimicing growth up a substrate.

${ }^{32}$ H. M. Nguyen, P.-Y. Hsiao, and M.-H. Phan, J. Appl. Phys. 107, 09D706 (2010).

${ }^{33}$ U. Nowak, in Handbook of Magnetism and Advanced Magnetic Materials, edited by H. Kronmüller and S. Parkin (John Wiley and Sons, New York, 2007), Vol. 1.

${ }^{34}$ V. Baltz, G. Gaudin, P. Somani, and B. Dieny, Appl. Phys. Lett. 96, 262505 (2010).

${ }^{35}$ K. D. Usadel and U. Nowak, Phys. Rev. B 80, 014418 (2009).

${ }^{36}$ M. Ali, P. Adie, C. H. Marrows, D. Greig, B. J. Hickey, and R. L. Stamps, Nat. Mater. 6, 70 (2007).

${ }^{37}$ T. Arbuzova, B. Gizhevskii, S. Naumov, A. Korolev, V. Arbuzov, K. Shalnov, and A. Druzhkov, J. Magn. Magn. Mater. 258, 342 (2003).

${ }^{38}$ M. Bode, E. Y. Vedmedenko, K. von Bergmann, A. Kubetza, P. Ferriani, S. Heinze, and R. Wiesendanger, Nat. Mater. 5, 477 (2006). 